\title{
¿QUÉ ES LO SUBNACIONAL DE LA DESIGUALDAD SUBNACIONAL? \\ UNA MIRADA INTERSECCIONAL A LA DESIGUALDAD EN \\ LATINOAMÉRICA*
}

\author{
What is the Subnational of Subnational Inequality? An Intersectional \\ Look at Inequality in Latin America
}

\author{
SILVIA OTERO-BAHAMÓN
}

Universidad del Rosario, Bogotá, Colombia

\begin{abstract}
RESUMEN
La investigación subnacional en América Latina ha establecido que en los países de la región las condiciones de vida están fuertemente determinadas por el lugar de residencia. Pero a pesar de estos avances, sabemos muy poco sobre cómo lo subnacional interactúa con otros atributos como género, raza y ruralidad para producir un paisaje diverso de desventajas y privilegios. Este artículo utiliza el enfoque de la interseccionalidad para mostrar las enormes diferencias en analfabetismo e inasistencia a escuela entre grupos compuestos por distintas combinaciones de dichos atributos en Perú, Colombia y Chile. Para entender mejor el efecto de la dimensión subnacional en esas diferencias, el artículo aplica la descomposición Oaxaca-Blinder e identifica qué porción de las brechas regionales se debe a distintas composiciones demográficas entre las regiones. Los anteriores análisis motivan una reflexión teórica sobre la naturaleza de la desigualdad subnacional que se basa en distinguir un componente composicional y otro interseccional e identificar posibles causales de ambos.
\end{abstract}

Palabras clave: desigualdad subnacional, interseccionalidad, analfabetismo, América Latina.

\begin{abstract}
Subnational research in Latin America has established that living conditions in the region's countries are strongly determined by place of residence. However, despite these advances, we know very little about how the subnational dimension interacts with other attributes such as gender, race, and rurality to produce a diverse landscape of disadvantage and privilege. This article uses the intersectionality approach to show the enormous differences in illiteracy and school non-attendance among groups composed of different combinations of these attributes in Peru, Colombia, and Chile. To better understand the effect of the subnational dimension on these differences, the article applies the Oaxaca-Binder decomposition and identifies what portion of the regional gaps is due to different demographic compositions across regions. This analysis motivates a theoretical reflection on the nature of subnational inequality based on distinguishing a compositional and an intersectional component and identifying possible causal factors of both.
\end{abstract}

Keywords: subnational inequality, intersectionality, illiteracy, Latin America.

Este artículo es producto del proyecto “ ¿Cómo se reduce la desigualdad en las ciudades colombianas? Política, Políticas, Economía y Suerte en Bucaramanga, Pereira, Barranquilla y Cartagena", financiado con recursos provenientes del Patrimonio Autónomo Fondo Nacional de Financiamiento para la Ciencia, la Tecnología y la Innovación Francisco José de Caldas bajo el contrato 174-2019.

Agradecimientos: la autora agradece a María Camila Herrera y Liney Álvarez por su trabajo en este artículo como asistentes de investigación.

DOI: $10.4067 /$ S0718-090X2021005000105 


\section{INTRODUCCIÓN: EL CALEIDOSCOPIO DE LA DESIGUALDAD EN AMÉRICA LATINA}

La pandemia del Covid-19 que suspendió al planeta desde inicios de 2020 desnudó la imposible convivencia de las naciones latinoamericanas con niveles alarmantes de desigualdad (Lustig y Mariscal 2020). Las cuarentenas y el cierre de actividades evidenciaron las enormes diferencias entre lugares, etnias, géneros, y ocupaciones sociales en cosas tan diversas como disponibilidad de internet, acceso a servicios de salud, agua potable en casa y seguridad alimentaria. El virus se encarnó con fuerza en algunos lugares y grupos sociales específicos como la población indígena del Amazonas de Colombia, Perú y Brasil, los barrios de inmigrantes en Santiago de Chile, y los asentamientos urbanos periféricos de Lima en Perú. El Covid-19 fue un evento dramático para el continente que no sólo demostró con crudeza por qué América Latina ranquea consistentemente como la región más desigual del planeta (Ábramo 2019; Lustig 2020), sino que evidenció las enormes diferencias entre grupos en la intersección de distintos atributos como género, etnia, región y ocupación.

Los académicos y hacedores de políticas públicas del continente han recorrido ya un camino evidenciando las desigualdades de raza, género y ruralidad que se viven en los distintos países (Wade 1997; Hoffman y Centeno 2003; Molinas Vega et al. 2011; Bertola y Williamson 2017; PNUD 2019; Busso y Messina 2020). Pero en años recientes la agenda de investigación sobre la dimensión subnacional ha sumado a esa evidencia previa para demostrar que el lugar de residencia dentro de un país también constituye una fuente de privilegio o desventaja (Otero-Bahamón 2016, 2019, 2020; Singh 2016, Giraudy y Pribble 2020; Harbers y Steele 2020). En general ser mujer, hacer parte de una minoría étnica, estar en el área rural y vivir en ciertas regiones obstaculizan el acceso a recursos y bienes públicos de buena calidad, conllevando a peores estándares en calidad de vida para las personas que pertenecen a dichas categorías. Pero estos cuatro atributos (género, raza, ruralidad y región) interactúan de formas complejas para producir desventajas o privilegios. Y además, el efecto de dichas combinaciones cambia de un país a otro y varía significativamente en el tiempo. Esta noción, que se conoce en la literatura como la interseccionalidad (Collins 2015), ha tenido una atención insuficiente en América Latina (Solis et al. 2019) y sabemos incluso menos sobre la interacción entre lo subnacional y otros atributos como género, raza y ruralidad para producir un paisaje diverso de desventajas y privilegios.

Este artículo parte de la premisa de Giraudy et al. (2019) según la cual la investigación subnacional permite comprender mejor fenómenos clave para la ciencia política y las ciencias sociales. En este caso, adoptar el lente subnacional -tanto teórica como metodológicamente-, ilumina aspectos de la desigualdad racial, de género y de clase que permanecían ocultos y que pueden ser relevantes para el diseño de la política social (Antía 2018). Como se hará evidente más adelante, el hecho que ciertas regiones agraven o atenúen la discriminación de 
género, raza, o clase, sugiere la importancia de agentes y procesos en lo subnacional para explicar la naturaleza de la desigualdad en la región. Pero más crucialmente, comprender el nivel, la variación y la naturaleza de la desigualdad subnacional es en sí mismo un importante motivo. Las páginas que siguen abordan estas preguntas aplicando el enfoque interseccional desde una metodología cuantitativa y descriptiva (Else-Quest y Hyde 2016; Bowleg y Bauer 2016; Bauer y Scheim 2019). Utilizando microdatos provenientes de las últimas dos rondas censales en Colombia (2005 y 2018), Perú (2007 y 2017), Chile (2002 y 2017), el estudio se desarrolla en dos momentos. En el primero realiza una caracterización de las diferencias en la prevalencia del analfabetismo según cuatro atributos importantes para la definición de la identidad y el acceso a recursos -género, etnia, condición de ruralidad, región y su intersección. Esta caracterización sirve varios propósitos. Primero, demuestra que cuando nos enfocamos en promedios nacionales o promedios para grandes categorías sociales como el género o la etnia enmascaramos importantes desigualdades que valen la pena atender y reducir. Segundo, cuantifica la magnitud de las brechas sociales existentes entre grupos y su evolución en el tiempo. Tercero identifica las intersecciones o las combinaciones de atributos en las que las personas están en un mayor -y menor- riesgo de sufrir discriminación y opresión, reflejada en menor acceso a educación. Finalmente, el ejercicio de la caracterización identifica cuáles son las regiones dentro de cada país que agravan las brechas de género, etnia o ruralidad (Bowleg y Bauer 2016; Nuru-Jeter et al 2018).

En un segundo momento, el artículo aplica la descomposición Oaxaca-Blinder para entender y reflexionar sobre la naturaleza de la desigualdad subnacional (Oaxaca 1973; Blinder 1973). Dicha metodología, que será explicada con profundidad más adelante, permite desglosar hasta qué punto la diferencia entre grupos -en este caso regiones- en un atributo -analfabetismo o inasistencia a escuela- se debe por diferencias en la composición de las regiones, o por diferencias en los efectos que tienen los elementos de dicha composición. Este punto es muy importante puesto que varias teorías establecen que las desigualdades subnacionales (o desigualdades regionales y espaciales como también se les denomina en la literatura) son causadas por la distribución desigual en el territorio de factores como recursos naturales, dinámicas de aglomeración espacial (Krugman 1991), la industrialización (Bértola 2020) o minorías étnicas (Dell 2010). Desde estas variadas e interdisciplinares perspectivas, procesos territorialmente heterogéneos de desarrollo económico, acumulación de capital, y/o racismo van haciendo que unas regiones tengan peores empleos, bienes públicos y servicios sociales. Esto va generando un impacto en la composición de dichas regiones, que con el tiempo tienen una mayor proporción de grupos tradicionalmente marginados como habitantes rurales y minorías étnicas. Se puede derivar de estas aproximaciones que la región o la unidad subnacional no tendría una fuerza causal independiente que produciría ventajas y o discriminaciones, sino que reflejaría los efectos del racismo, el sesgo urbano y los modelos de desarrollo económico que se concentran en su territorio. 
En contraste con estas teorías, otras teorizan la desigualdad subnacional no como expresión espacial de otros tipos de desigualdades, sino como producto de las instituciones mismas que dividen el país en unidades políticas y administrativas como estados o departamentos. Según estas perspectivas, instituciones ancladas en las divisiones subnacionales tales como el federalismo, las transferencias interregionales, el malapportionment, y los sistemas de partidos centrífugos contribuyen a espacializar la discriminación y a producir diferenciaciones espaciales en el desarrollo económico y social (Beramendi 2012; Rogers 2021).

La descomposición Oaxaca-Blinder nos permite darles cabida a ambas inclinaciones teóricas. La porción explicada, que se eliminaría si los dos grupos tuvieran la misma composición, se puede denominar la porción composicional, mientras que la porción no explicada, que incluye el efecto heterogéneo que ser mujer o de una minoría étnica tiene en las distintas regiones, podría denominarse la porción interseccional de la desigualdad subnacional. Este artículo analiza los dos componentes para concluir que en ambos opera una dimensión subnacional de la desigualdad. Sin embargo, este efecto subnacional no es constante, sino que cambia de un país a otro y varía significativamente en el tiempo.

Con esta aplicación cuantitativa y descriptiva de la investigación subnacional y la teoría de la interseccionalidad, este estudio constituye un aporte original para la comprensión de la desigualdad en América Latina y el rol de la dimensión subnacional en ella. En mi conocimiento éste es el primer estudio que aterriza el enfoque interseccional utilizando las últimas rondas censales para describir evolución en las diferencias entre grupos compuestos por la intersección de varios atributos, y constituye una contribución novedosa a la conceptualización de la desigualdad subnacional.

\section{LA TEORÍA DE LA INTERSECCIONALIDAD Y SU APLICACIÓN EN AMÉRICA LATINA}

El concepto de interseccionalidad se refiere a la interacción entre atributos o marcadores de la identidad que producen experiencias de vida de privilegio u opresión (Gopaldas 2013). Como establece Collins (2015), bajo este enfoque, aspectos de la identidad o del contexto como la raza, clase, el género, la orientación sexual, el origen étnico, la nacionalidad, las condiciones de discapacidad, y la edad -entre otras- no operan como entidades unitarias y mutuamente excluyentes, sino que son fenómenos que se construyen y determinan recíprocamente para dar forma a desigualdades sociales.

El concepto de interseccionalidad fue acuñado inicialmente por Crenshaw (1991) para evidenciar que la experiencia de las mujeres afrodescendientes era fundamentalmente distinta a la de las mujeres por un lado y de los afrodescendientes por otro. La intersección alude así a una noción multiplicativa y no 
aditiva de la desigualdad, pues, en otras palabras, las mujeres negras -para continuar el ejemplo-sufren una opresión mayor a la de la suma de ser mujeres y negras por separado.

En las últimas décadas el concepto de interseccionalidad ha viajado desde el feminismo negro para aplicarse a otros contextos y literaturas como la salud pública (Bowleg 2012; Evans et al 2018), la psicología (Rosenthal 2016), las relaciones internacionales (Krizsan et al 2012) y el mercado laboral (Browne y Misra 2003). Además del género y la raza se han evidenciado los efectos interactivos de otros marcadores como la clase social, origen nacional y orientación sexual entre otros. La interseccionalidad parte de la idea que la importancia de los marcadores varía con respecto al lugar y el tiempo. La raza, por ejemplo, es clave para estructurar las relaciones de poder, el acceso a recursos y las experiencias de vida en los Estados Unidos, pero no tiene tal importancia en otros contextos. El enfoque apunta entonces a no privilegiar un marcador determinado, sino a explorar en cada contexto los aspectos que puedan ser constitutivos para las relaciones de poder.

A pesar del enorme potencial del enfoque interseccional, y de la larga vigencia del término, su adopción es todavía incipiente en las ciencias sociales y subsisten importantes vacíos. Por un lado, no es común tener en cuenta el lugar de residencia dentro de un país como categoría social que interactúa con otras en la generación de desigualdades. ${ }^{1}$ Por otro, el enfoque y en particular sus aplicaciones cuantitativas han tenido una aplicación muy limitada a los análisis de desigualdad en América Latina. ${ }^{2}$ Este artículo aspira a contribuir en la superación de ambos vacíos.

\section{METODOLOGÍA PARA EL ENFOQUE INTERSECCIONAL}

Una de las razones por las que el enfoque interseccional ha tenido una aplicación limitada es porque no ha sido fácil identificar un método que cristalice las aspiraciones ontológicas del término (Hancock 2007; Bauer 2014; Collins 2015).

Inicialmente este enfoque era casi exclusivamente aplicado empíricamente en estudios de corte cualitativo donde etnografías y estudios de caso detallados se enfocaban en un número limitado de grupos interseccionales. Estas metodologías facilitaban el enfoque interseccional porque permitían identificar más claramente las categorías sociales que determinaban las experiencias de vida y los procesos sociales que conllevaban a discriminación o privilegio (Evans 2019). Esto ha llevado a algunos autores a afirmar que la metodología cualitati-

\footnotetext{
Algunos estudios que incorporan el aspecto subnacional o espacial en el estudio de la interseccionalidad son Malmusi et al. (2010); Scott y Siltanen (2012); Spierings (2016); Hopkins (2019); Rodó-de-Zárate y Baylina (2018).

Algunas aplicaciones cualitativas son Boesten (2010); Shannon et al. (2017); Caro Molina (2017); Baquero Melo (2017); Babb (2017). En cuanto a cuantitativas Mora (2019) y Kabeer y Santos (2017).
} 
va está mejor diseñada para examinar la interseccionalidad. Pero en la medida en la que ha aumentado la acogida del enfoque interseccional, se ha abierto la puerta a estudiarla desde un enfoque cuantitativo, y así mismo se ha discutido ampliamente qué método permite hacer una correspondencia entre la teoría y los supuestos del modelamiento estadístico.

Los enfoques cuantitativos aportan a la interseccionalidad porque ayudan a identificar atributos o características de las personas o del contexto que resultan influyentes en la asignación de privilegios o recursos y que no estaban previamente en el radar de los investigadores. También transmiten la magnitud y la distribución de desigualdades socio-estructurales, e identifican los procesos causales que afectan dicha diferenciación y evolución en el tiempo (Bowleg y Bauer 2016).

No obstante, las ganancias de adoptar metodologías cuantitativas no vienen sin enormes desafíos. El más importante de ellos ha sido identificar un método que traslade a las variables explicativas una lógica interseccional y no unitaria, y que logre en el modelamiento estadístico una noción multiplicativa y no aditiva de sus efectos. Los modelos tradicionales de regresión lineal tienen una lógica unitaria y aditiva, mientras que la introducción de términos de interacción no resuelve el problema de la aditividad. El apéndice incluye un resumen de las metodologías cuantitativas típicamente utilizadas en el estudio de la interseccionalidad.

Las limitaciones de los métodos más usados han llevado a adoptar otro tipo de modelos, tales como las descomposiciones de los efectos o los modelos multinivel. En este artículo adoptamos la primera alternativa porque nos permite reflexionar teóricamente sobre la naturaleza de la dimensión subnacional como categoría constitutiva de desigualdad.

\section{Descomposición Oaxaca Blinder como método para la interseccionalidad}

Parafraseando a Sen (2014), la descomposición Oaxaca-Blinder (Oaxaca 1973; Blinder 1973) consiste en descomponer con ayuda de regresiones la brecha en una variable de interés $(Y)$ entre dos grupos en un componente "explicado" y otro "no explicado". "La parte explicada de la brecha es la diferencia en la variable de interés atribuible a diferencias en los niveles de las variables predictoras $(X)$ entre el grupo "aventajado" y el grupo "desaventajado". Es decir que se refiere a la forma como se componen los grupos. La parte "no explicada" emerge de tres fuentes: a) diferencias en cómo las variables predictoras están asociadas con la variable dependiente en los dos grupos", es decir, de diferencias en los coeficientes; b) otros factores o variables omitidas que afectan el desenlace solo en uno de los grupos; c) error de medición de las variables sistemáticamente diferente entre los dos grupos (Sen 2014). Mientras que la parte explicada se podría corregir si la composición o la distribución de dotaciones del grupo marginado fuera la misma que del grupo privilegiado (Jackson y VanderWeele 2019), la "no explicada" "persistiría incluso si el grupo marginado lograra los mismos niveles de las variables predictoras del grupo privilegiado" (Sen 2014). Una de 
las posibles razones que explicaría esta parte no explicada es la discriminación, pues ésta haría que los mismos atributos conllevaran a efectos distintos. ${ }^{3}$

La descomposición Oaxaca-Blinder es una metodología cuantitativa apropiada para el estudio de la interseccionalidad (Jackson y VanderWeele 2019), en especial cuando se tiene en cuenta la dimensión subnacional. En nuestro caso, y como se explicará más adelante, al descomponer la diferencia en analfabetismo (o inasistencia a escuela en el caso de Chile) entre la región más privilegiada y la más marginada, una porción de la parte "no explicada" corresponde a los efectos heterogéneos que tiene la interacción de categorías y atributos en cada contexto regional, mientras que la parte explicada corresponde a las diferentes composiciones de los grupos.

\section{Metodología de este estudio}

Con base en la discusión anterior, este artículo utiliza microdatos de las dos últimas rondas censales realizadas entre 2002 y 2018 en Colombia, Perú y Chile para identificar la distribución de indicadores de educación entre grupos conformados por distintas combinaciones de género, etnia, condición de ruralidad y región.

Hago enfoque en estos tres países ya que los tres comparten varias características que son importantes para la provisión de bienes públicos. Los tres son países unitarios de mediano tamaño, con importante presencia de minorías étnicas en algunos territorios, con olas censales que coinciden en el tiempo y niveles de desarrollo económico intermedios. A pesar de las similitudes los tres presentan niveles diferentes de analfabetismo o inasistencia a escuela. Chile tiene menor analfabetismo y mejor sistema educativo, Colombia está en un nivel intermedio, y Perú excluye a importantes porciones de la población. En los tres países la cuestión regional es importante.

Me interesa explorar el impacto de distintos atributos en analfabetismo puesto que éste sigue siendo un problema importante para varios países de la región. El alfabetismo se define "como la capacidad de comprender, evaluar, usar e interactuar con textos escritos para participar en la sociedad, lograr los objetivos propios y desarrollar el conocimiento y la potencialidad individual" (Vágvölgyi et. al 2016). Sin las habilidades básicas de saber leer y escribir se dificulta el mejoramiento de la calidad de vida, el acceso a mejores empleos, el ejercicio de la ciudadanía, y el goce de la salud. Por ello no es casualidad que uno de los determinantes más poderosos de la pobreza sea no saber leer y escribir (Martínez et. al 2014). América Latina ha avanzado sustancialmente en la eliminación de esta condición, y hoy se estima que esta afecta a 9\% de la población adulta (Martínez et. al 2014: 6). No obstante, y como mostraremos, esta figura no es igual para todos los grupos sociales. Al impactar tanto la calidad de vida, se hace necesario entender cómo el analfabetismo 
afecta más a ciertos grupos que a otros. Finalmente, el analfabetismo es generalmente registrado por los censos de población, haciendo más fácil la realización de análisis comparativos entre momentos y países.

Decidí agregar los departamentos (Perú, Colombia) y regiones (Chile) en un puñado de regiones que tuviera sentido según la forma como tomadores de decisiones y cientistas sociales históricamente han conceptualizado el territorio. Esto permitió no sólo identificar diferencias que tienen sentido en los contextos locales, sino también simplifican el análisis. Debido a que para cada unidad subnacional se identificarían entre 8 y 12 estratos, no era factible hacer ese ejercicio para todas las unidades subnacionales de primer orden de cada país. Las decisiones sobre cómo agregar los departamentos fueron informadas por la forma como tradicionalmente se conciben las regiones en los tres países. Para Perú se utilizó la clásica división Costa, Sierra y Selva en la que se agregan las estadísticas oficiales. Para Colombia se identificaron las regiones naturales y se agregaron los departamentos entre la región Caribe, Orinoquía-Amazonía, Pacífica y Andina, que al ser la región más poblada se dividió en dos: Andina Central y Oriental. Para Chile se utilizó la clasificación tradicional entre Norte ampliado, Centro y Sur.

Para la primera ronda censal de los tres países (2002 en Chile, 2005 en Colombia, 2007 en Perú) acudí a los microdatos censales procesados por IPUMS (Minnessota Population Center 2019) que maneja una muestra censal representativa del 10\% de la población con factores de expansión que generan información sobre el total de la población. Para la segunda ronda censal (2017 en Chile y Perú y 2018 en Colombia) utilicé los microdatos censales del total de la población obtenidos directamente con la agencia estadística de cada país. La lista de variables incluidas en cada censo se encuentra en la Tabla 1.

Tabla 1. Censos y Variables.

\begin{tabular}{|c|c|c|c|}
\hline País & $\begin{array}{l}\text { Censos y } \\
\text { fuente }\end{array}$ & $\begin{array}{c}\text { Inclusiones y exclusio- } \\
\text { nes }\end{array}$ & Regiones \\
\hline Colombia & $\begin{array}{l}\text { 2005: IPUMS y } \\
\text { DANE } \\
\text { 2018: DANE }\end{array}$ & $\begin{array}{l}\text {-Departamento } \\
\text {-Género (Femenino y } \\
\text { Masculino) } \\
\text {-Pertenece a algún grupo } \\
\text { étnico (Ninguno, Indíge- } \\
\text { na, Negro-Afrocolombia- } \\
\text { no-Raizal-Palenquero) } \\
\text {-Área de residencia (ur- } \\
\text { bano/rural) } \\
\text {-Sabe leer y escribir (si/ } \\
\text { no) } \\
\text {-Edad (mayores de } 15 \\
\text { años) }\end{array}$ & $\begin{array}{l}\text { Caribe }{ }^{4} \\
\text { Atlántico, Cesar, Córdoba, Bolívar, Mag- } \\
\text { dalena, Guajira, San Andrés Islas y Su- } \\
\text { cre. } \\
\text { Andina Central: } \\
\text { Antioquia, Huila, Tolima, Boyacá, Cun- } \\
\text { dinamarca, Meta, Bogotá D. C., Caldas, } \\
\text { Quindío y Risaralda. } \\
\text { Oriental: } \\
\text { Arauca, Casanare, Norte de Santander, } \\
\text { Santander. } \\
\text { Pacífica: } \\
\text { Chocó, Cauca, Valle del Cauca y Nariño. } \\
\text { Amazonía-Orinoquía: } \\
\text { Amazonas, Caquetá, Guaviare, Guainía, } \\
\text { Putumayo, Vaupés y Vichada. }\end{array}$ \\
\hline
\end{tabular}

Para Colombia se usaron como referencia las Regiones Administrativas de Planificación (RAP) pero se hicieron algunas modificaciones. 


\begin{tabular}{|c|c|c|c|}
\hline País & $\begin{array}{l}\text { Censos y } \\
\text { fuente }\end{array}$ & $\begin{array}{c}\text { Inclusiones y exclusio- } \\
\text { nes }\end{array}$ & Regiones \\
\hline Perú & $\begin{array}{l}\text { 2007: IPUMS e } \\
\text { INEI } \\
\text { 2017: INEI }\end{array}$ & $\begin{array}{l}\text {-Departamento } \\
\text {-Género (Femenino y } \\
\text { Masculino) } \\
\text { - Lengua aprendida en } \\
\text { la niñez (lenguas indíge- } \\
\text { nas/castellano) } \\
\text {-Área de residencia (ur- } \\
\text { bano/rural) } \\
\text {-Sabe leer y escribir (si/ } \\
\text { no) } \\
\text {-Edad (mayores de } 15 \\
\text { años) }\end{array}$ & $\begin{array}{l}\text { Costa: } \\
\text { Lima, La Libertad, Piura, Ica, Lambaye- } \\
\text { que, Callao, Tumbes, Tacna, Moquegua. } \\
\text { Sierra: } \\
\text { Cusco, Pasco, Ayacucho, Huanuco, Ju- } \\
\text { nin, Cajamarca, Áncash, Arequipa, Apu- } \\
\text { rimac, Huancavelica y Puno. } \\
\text { Selva: } \\
\text { San Martín, Ucayali, Loreto, Madre de } \\
\text { Dios, Amazonas }\end{array}$ \\
\hline Chile & $\begin{array}{l}\text { 2002: IPUMS e } \\
\text { INE } \\
\text { 2017: INE }\end{array}$ & $\begin{array}{l}\text {-Provincia } \\
\text {-Género (Femenino y } \\
\text { Masculino) } \\
\text {-Pertenece a grupo indí- } \\
\text { gena } \\
\text {-Área de residencia (ur- } \\
\text { bano/rural) } \\
\text {-Años de escolaridad } \\
\text {-Edad (mayores de } 15 \\
\text { años) }\end{array}$ & $\begin{array}{l}\text { Norte: } \\
\text { Regiones XV, I, II, III y IV. } \\
\text { Central: } \\
\text { Regiones V, VI, VII, XVI y Santiago. } \\
\text { Sur: } \\
\text { Regiones VIII, IX, X, XI, XII y XIV. }\end{array}$ \\
\hline
\end{tabular}

En Chile no se pudo realizar el análisis de la variable analfabetismo porque el Censo de 2017 no preguntó a los chilenos si sabían leer y escribir. Para subsanar esta dificultad tomamos la decisión de calcular el porcentaje de personas mayores de 15 años con 4 años o menos de escolaridad, ya que el primer ciclo de educación básica en Chile consiste en cuatro años.

A partir de la combinación de las categorías relacionadas con región, género, etnicidad y área se crearon intersecciones o estratos y se calculó la tasa de analfabetismo (o ausencia de más de 4 años de escolaridad) para cada uno. En Colombia se obtuvieron 5 regiones (Andina Central, Oriental, Pacífica, Amazonía-Orinoquía y Caribe), 2 géneros (Mujer-Hombre), 3 categorías étnicas (Indígena, Afrodescendiente, Ninguno) y 2 áreas (Urbano-Rural) que al ser combinadas dieron lugar a 60 intersecciones o estratos. En Perú se obtuvieron 3 regiones (Costa, Sierra y Selva), 2 géneros (Mujer-Hombre), 2 categorías étnicas (Lengua Indígena-Castellano) y 2 áreas (Urbano-Rural) que al ser combinadas dieron lugar a 24 intersecciones o estratos. En Chile se obtuvieron 3 regiones (Norte, Central, Sur), 2 géneros (Mujer-Hombre), 2 categorías étnicas (Indígena - No indígena) y 2 áreas (Urbano-Rural) que al ser combinadas dieron lugar a 24 intersecciones o estratos. Adicionalmente se calculó la tasa de analfabetismo (o de ausencia de 4 años o menos de escolaridad) para las categorías en agregado.

Se decidió utilizar esta variable y no la de pertenencia a grupo étnico porque ésta sólo se incluyó en el Censo de 2017 y no en el de 2007, impidiendo la comparabilidad. La lengua aprendida en la niñez es un buen proxy de pertenencia étnica. 
Los resultados se discuten en la siguiente sección.

\section{EL ANALFABETISMO SEGÚN RAZA, GÉNERO, REGIÓN Y RURALIDAD Y SU INTERSECCIÓN}

La expansión de la educación básica es América Latina es una de las políticas públicas que más ha impactado la reducción de la pobreza y la desigualdad (Lopez-Calva y Lustig 2010). Y si bien los avances han sido importantes, cuando miramos las diferencias de género, etnia, área y región subsisten importantes diferencias.

Como se observa en la gráfica 1, el analfabetismo se redujo entre los 2000s y los 2010s: pasó del 7\% al 5.7\% en Perú y del 8\% al 5\% en Colombia. En Chile el porcentaje de personas con cuatro años o menos de escolaridad se redujo de casi $14 \%$ al $8 \%$. Pero este promedio nacional relativamente bajo para todos los países enmascara importantes variaciones y desigualdades.

Gráfica 1. Tasas nacionales de Analfabetismo (Colombia - Perú) y Porcentaje de personas con cuatro años o menos de escolaridad (Chile).

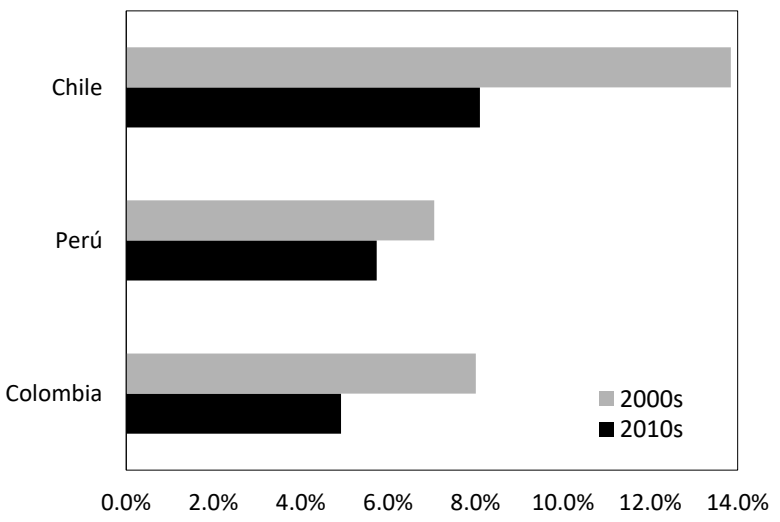

Cuando adoptamos el enfoque de género (Gráfica 2) vemos que hay importantes diferencias en Perú mas no en Chile o Colombia. En Perú en 2007 las mujeres tenían una tasa 7 puntos porcentuales (p.p. de ahora en adelante) mayor que la de los hombres (10.5\% y 3.5\%). En 2017 la brecha se disminuyó a 5.5 p.p. $(8.4 \%$ y $3 \%)$. En Colombia la tasa de analfabetismo de las mujeres es ligeramente inferior que la de los hombres en ambas rondas censales. En Chile la ventaja de los hombres es de poco más de 1 p.p. 
Gráfica 2. Tasas nacionales de Analfabetismo (Colombia - Perú) y Porcentaje de personas con cuatro años o menos de escolaridad (Chile) según género

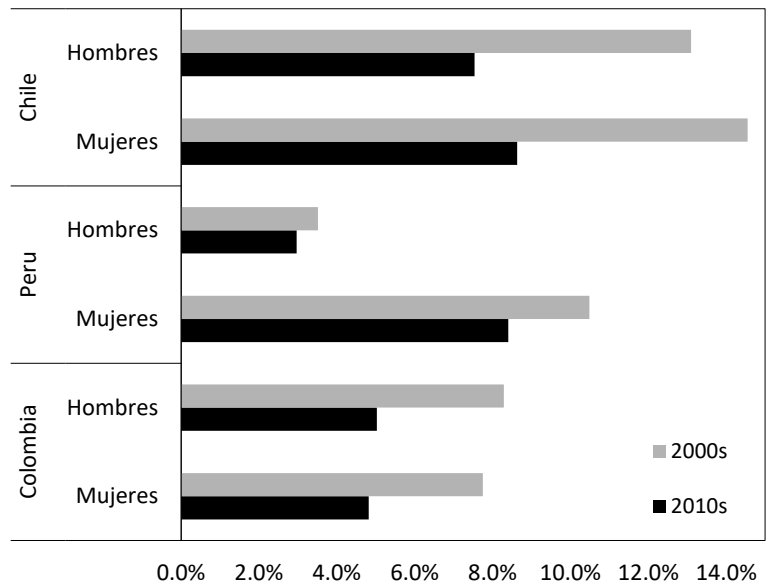

Al introducir el elemento étnico (Gráfica 3) observamos que los grupos indígenas tienen mucha mayor prevalencia de analfabetismo/no escolaridad que el resto y que la reducción ha sido importante en ese grupo. En Perú el analfabetismo en personas con lengua materna indígena es tres veces mayor que para el país en general y cinco veces mayor que para los que hablan castellano (21\% en 2007 y 16\% en 2017). En Colombia el analfabetismo entre los indígenas era casi cuatro veces el del promedio nacional en 2005 y 3.5 veces en 2017, con tasas del $30 \%$ y $17 \%$ respectivamente. En cuanto a los afrodescendientes, la diferencia entre ellos y los que no se identifican con ninguna etnia era de 5 p.p. en 2005 y 3.6 p.p. en 2017. En Chile la diferencia entre indígenas y no indígenas es menor. La porción de miembros de grupos indígenas que tenían cuatro años o menos de escolaridad es menos del doble que de los no indígenas en ambos años.

Gráfica 3. Tasas nacionales de Analfabetismo (Colombia - Perú) y Porcentaje de personas con cuatro años o menos de escolaridad (Chile) según componente étnico

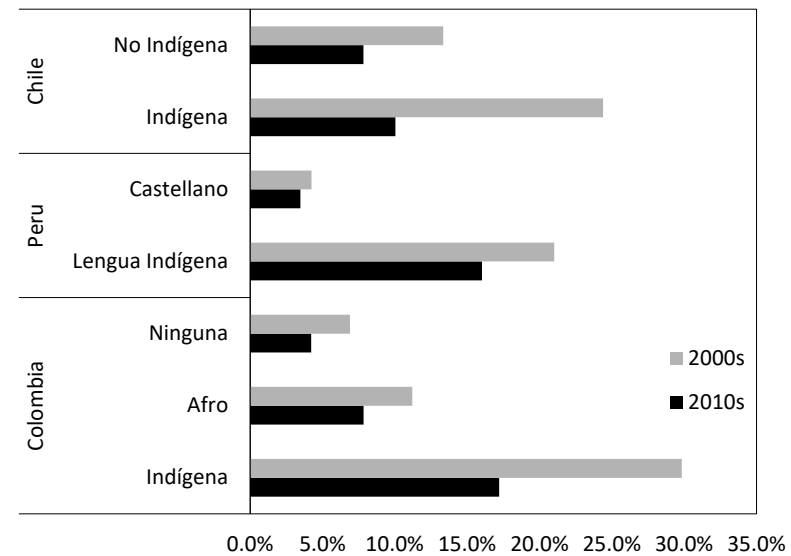


Una de las diferencias más significativas en la región es la que se da entre el campo y la ciudad (Gráfica 4). En Perú el analfabetismo rural es 5.4 veces superior que el urbano en ambos años, y la reducción en 10 años es insignificante, pues la tasa pasó del 19.6\% al 17.9\%. En Colombia el analfabetismo rural es 3.8 veces el del urbano en ambos años, pero la reducción ha sido más significativa, pues éste pasó del 18.8\% al 11.7\%. En Chile el porcentaje de los que no han tenido educación básica en el sector rural es 2.6 veces el del sector urbano en 2007 y 2.3 veces en 2017. La diferencia urbano-rural es menor en Chile que en los otros países y la magnitud de la brecha se ha reducido mientras que en los otros se ha mantenido constante.

Gráfica 4. Tasas nacionales de Analfabetismo (Colombia - Perú) y Porcentaje de personas con cuatro años o menos de escolaridad (Chile) según área rural y urbana.

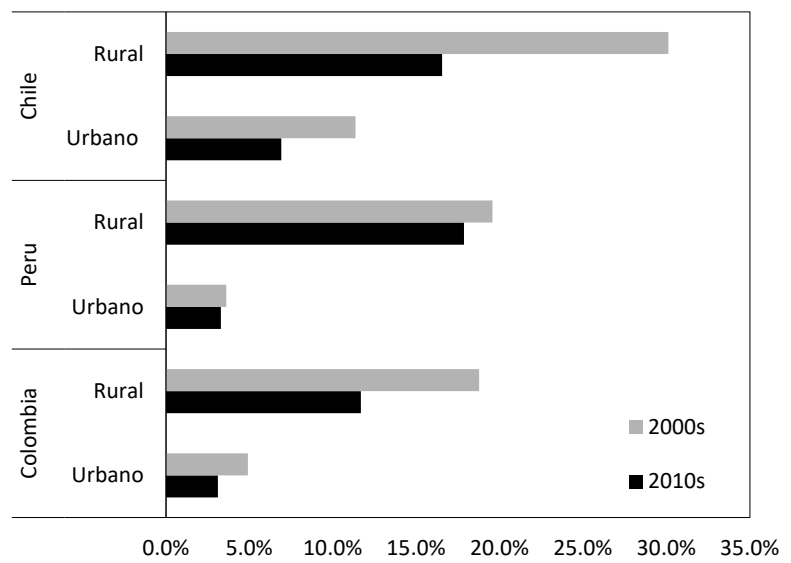

Finalmente, cuando adoptamos el lente regional (Gráfica 5) observamos algunas diferencias, aunque menores que las de área urbana-rural, etnia y género. En Perú el analfabetismo de la región Selva triplica el de la región Costa en ambas rondas censales, mientras que el de la región Sierra lo duplica. En Colombia la región Oriental, Amazonía-Orinoquía y Pacífica muestran tasas de analfabetismo similares. La brecha es más visible al comparar la región Caribe con la Andina Central pues tiene tasas de analfabetismo 2.5 veces superiores. En Chile la región Central y Norte tienen tasas de escolaridad similares, mientras que en el Sur se hace más visible la desventaja, al tener un porcentaje de baja escolaridad 1.5 veces superior que el de la región Central. 
Gráfica 5. Tasas nacionales de Analfabetismo (Colombia - Perú) y Porcentaje de personas con cuatro años o menos de escolaridad (Chile) según región.

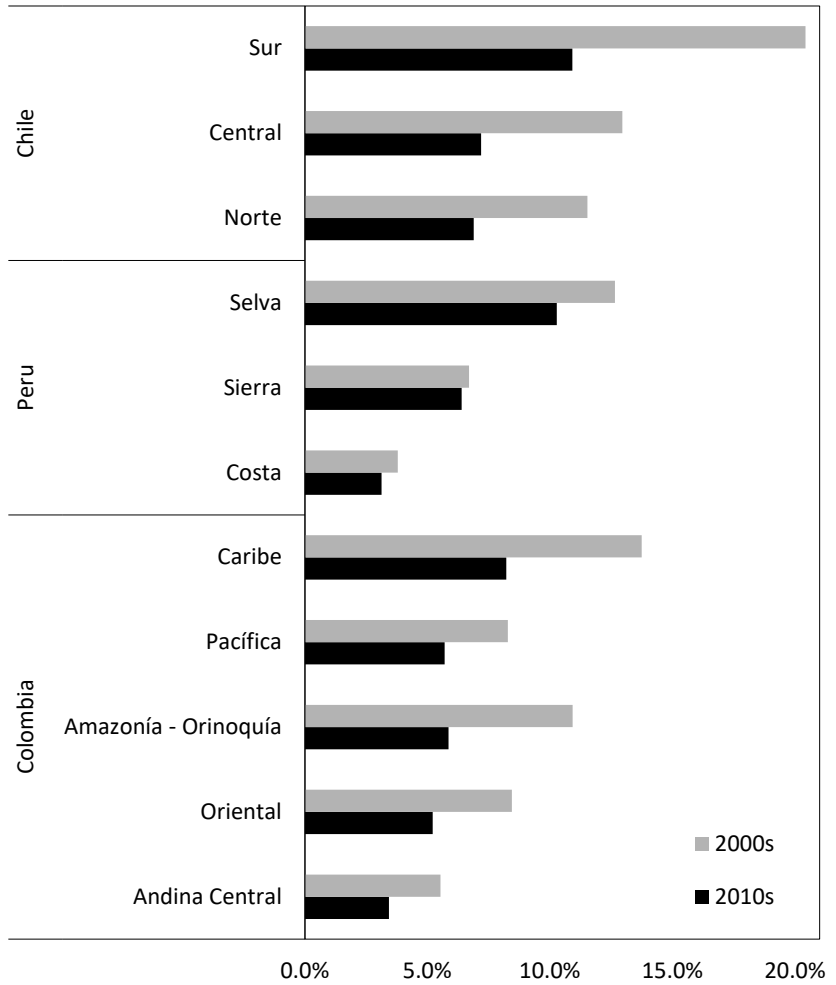

El análisis de cada categoría por separado nos muestra que en los países de América Latina los promedios esconden importantes diferencias entre géneros, etnias, condición urbana-rural y región. También evidencia que no todos sufren la misma severidad de brechas en las mismas categorías. En Colombia, por ejemplo, la brecha de género es mínima, y la brecha étnica es mayor que la de área urbano-rural. En Perú, en cambio, la brecha de género es más importante, y la categoría donde se registra una brecha de mayor tamaño es la urbano-rural. En Chile se registran brechas menos amplias, aunque las diferencias entre grupos indígenas y no indígenas, y entre la zona urbana y la rural son importantes. Todos los países tienen brechas regionales, pero estas no son más profundas que las de las otras categorías.

\section{La intersección de raza, región, género y ruralidad}

¿Qué pasa cuando cruzamos estos atributos? ¿Qué encontramos cuándo comparamos el analfabetismo o la escolaridad entre intersecciones de estas múltiples categorías? ¿La experiencia de todas las personas rurales o indígenas es la misma? ¿O varía por región y sexo? Tras calcular la tasa de analfabetismo-escolaridad en cada una de las intersecciones o estratos (60 para Colombia y 24 
para Chile y Perú) las ranqueamos de menor a mayor. La gráfica 6, gráfica 7 y gráfica 8 muestran el resultado de dicho ejercicio. Los párrafos que siguen analizan comparativamente los resultados según cuatro ejes de análisis: los grupos más privilegiados, los grupos más desventajados, las intersecciones a partir de género, etnia, ruralidad y región.

Gráfica 6. Analfabetismo según intersección de región - género - etnia - área en Colombia.

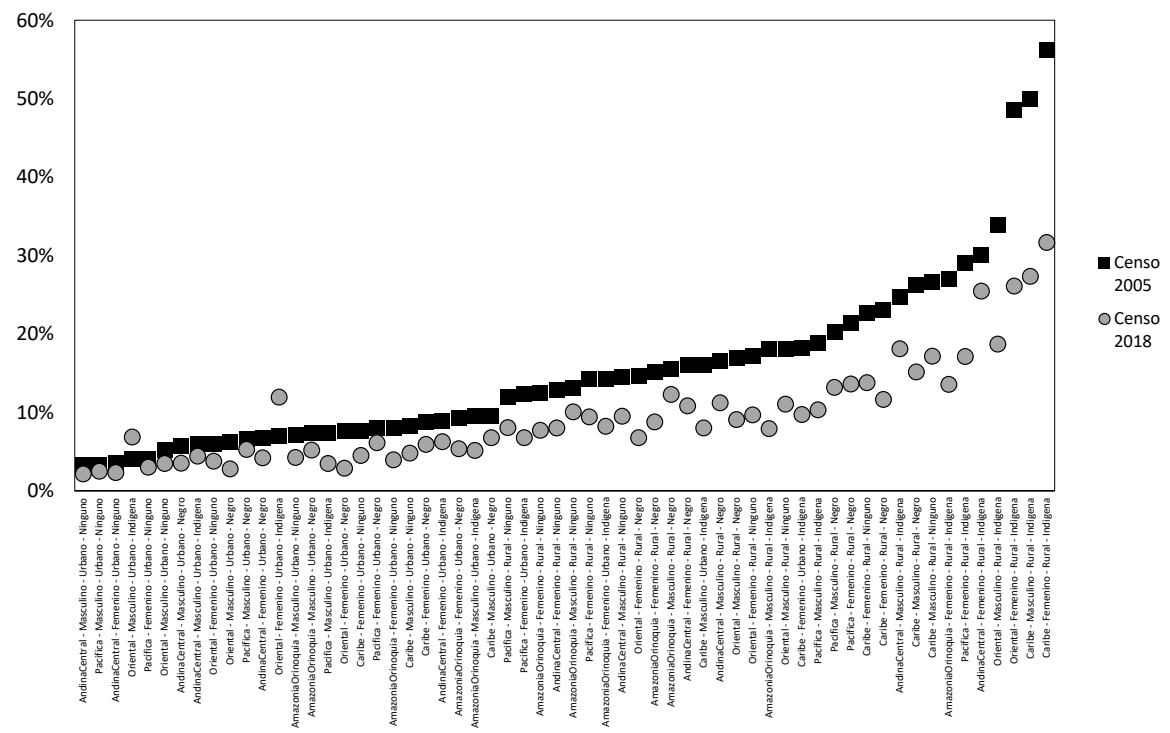

Gráfica 7. Analfabetismo según intersección de región - género - etnia - área en Perú.

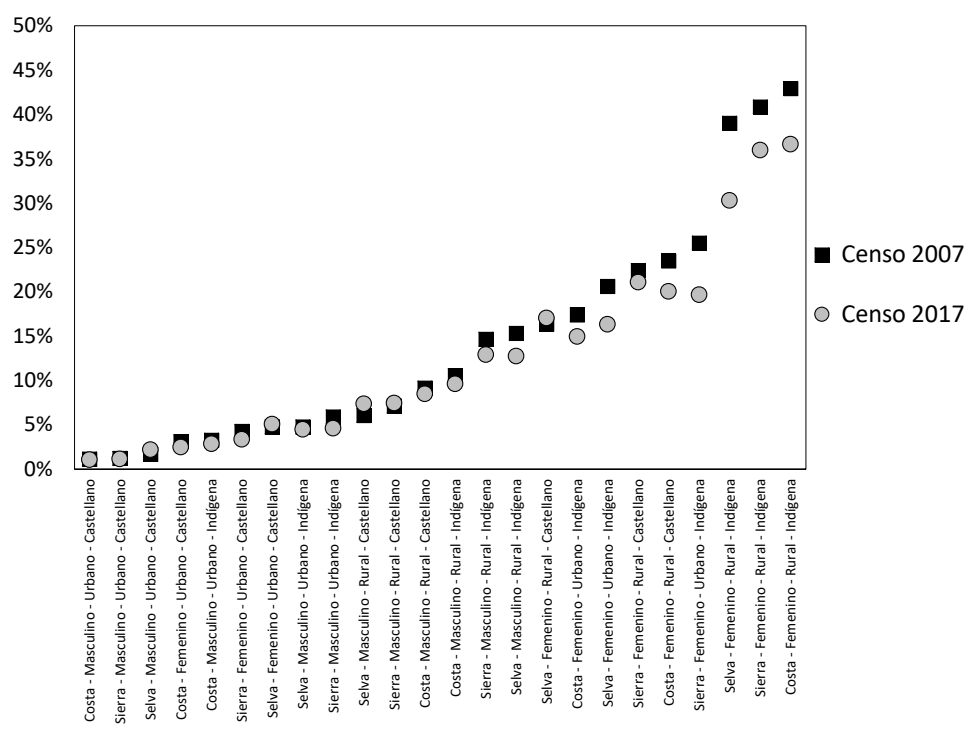


Gráfica 8. Porcentaje con 4 años o menos de escolaridad según intersección de región - género - etnia - área en Chile.

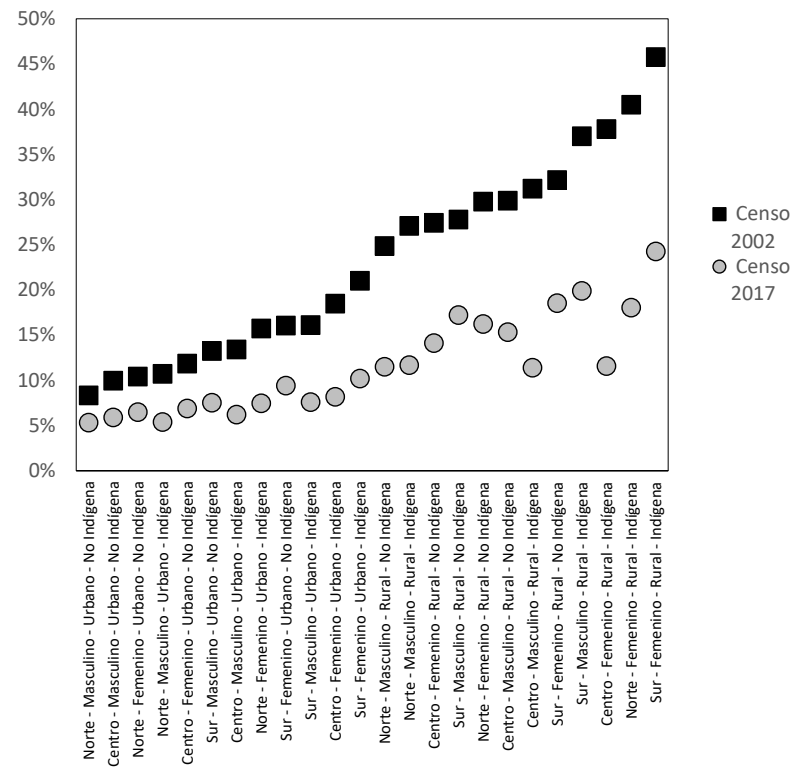

Como muestran las gráficas 6,7 y 8, existe una enorme dispersión entre estratos o intersecciones de categorías sociales incluso en indicadores en el que los países de la región han tenido una importante reducción. En Colombia para 2005 la tasa de analfabetismo varía entre el 3.33\% para hombres urbanos sin grupo étnico identificado de la región Andina central y 56.27\% para mujeres rurales indígenas de la región Caribe. Trece años después el rango de variación se redujo a $2.2 \%$ y $31.7 \%$ para los mismos grupos. En Perú la variación es mayor pues el analfabetismo en 2007 oscila entre 1.2\% para los hombres urbanos de la Costa que aprendieron castellano y el $43 \%$ para las mujeres rurales indígenas de la Costa. 10 años después el rango se redujo muy poco, pues mientras que el grupo privilegiado se mantuvo igual, el más desventajado presentó una tasa de analfabetismo de $36.6 \%$. En Chile en 2007, el porcentaje de personas con 4 años o menos de escolaridad varió entre $8.3 \%$ para hombres urbanos no indígenas del Norte y 46\% para mujeres rurales indígenas del Sur. En 2017 la brecha tuvo una importante reducción entre $5.3 \%$ para el primer grupo y $24.3 \%$ para el segundo. Los tres países muestran importantes desigualdades entre grupos y diferentes ritmos de reducción de brechas: mayores para Chile, menores para Perú, e intermedias para Colombia. Y si bien la causa de estos ritmos diferentes en la reducción de las brechas constituye una relevante pregunta de investigación, responderla está fuera del objetivo de este trabajo.

En Colombia hombres y mujeres rurales indígenas componen los 5 estratos con mayor analfabetismo, en Perú los cinco estratos con peor tasa de analfabetismo son de mujeres, 4 de ellos rurales y 4 indígenas. Y en Chile las mujeres rurales indígenas también constituyen 4 de los 5 estratos con menor escolaridad. En los 
distintos países ser mujer, indígena y rural constituye una condición de mucha desventaja.

Llama mucho la atención que para este sub-grupo en desventaja, la región hace una importante diferencia en Colombia, no tan importante en Chile y casi nula en Perú. La tasa de analfabetismo de mujeres rurales indígenas en Colombia oscila entre $14,6 \%$ y $31 \%$; $38 \%$ y $46 \%$ en Chile y $39 \%$ y $43 \%$ en Perú, con datos de la última ronda censal.

Mirando al otro lado del panorama, el grupo más privilegiado se compone de personas urbanas que no se identifican como miembros de minorías. En Colombia y en Chile hay mejor balance de género entre los cinco grupos más privilegiados, mientras que en Perú casi todos los grupos con menor tasa de analfabetismo son de hombres. En Colombia, y a diferencia de lo que ocurre con el grupo en mayor desventaja, para los más privilegiados la región no hace mayor diferencia: entre personas urbanas no indígenas la variación entre regiones es de 5 p.p. para 2005 y 3 p.p. para 2018. En Chile la variación regional para los más privilegiados es 6 p.p. para 2002 y 3 p.p. para 2017. En Perú es casi nula, entre 1 y 2 p.p.

\section{Las intersecciones con enfoque de género}

El análisis realizado también revela que hay algunas intersecciones que agravan (o neutralizan) las diferencias de género. Como recordaremos de la sección anterior, en el agregado nacional solo se observa diferencias de género en Perú, pues en Colombia y en Chile el analfabetismo o escolaridad es similar entre géneros. Sin embargo, cuando introducimos las otras categorías saltan a la vista otras tendencias. En Colombia son notorias las brechas de género entre los indígenas, y sobre todo a nivel rural: entre 6 y 15 p.p. para 2005 y 4 y 8 p.p. para 2018, dependiendo de la región. El género es una condición de discriminación para educación en el área rural entre los indígenas, y particularmente en la región Oriental.

En Perú, el área urbana y haber aprendido castellano como lengua materna atenúan la brecha de género de 7 p.p. (2007) y 5.5 p.p. (2017) observada en el agregado nacional, pues la diferencia entre hombres y mujeres es de solo 1 a 3 p.p. (dependiendo de la región). La brecha de género se empieza a notar en los castellano-hablantes del sector rural: en ambas rondas censales los hombres llevan una ventaja a las mujeres entre 10 y 15 p.p. dependiendo de la región. Ya entre los indígenas urbanos las brechas de género se profundizan un poco más: en 2007 la diferencia era entre 14 y 20 p.p. y en 2017 entre 12 y 15 p.p. según región. Finalmente, al igual que en Colombia el grupo de indígenas rurales es el que muestra las brechas más profundas de género, pero en mucha mayor magnitud que en el primer país analizado. En 2007 las diferencias entre mujeres y hombres con estas características oscilaban entre 25 y 32 p.p. según la región, y en 2017 oscilaban entre 18 y 26 p.p., siendo la Costa la región que más profundiza este tipo de brecha. Salta a la vista que las brechas de género prácticamente no se han recortado con el paso de los años. 
En Chile, como en los otros países, las brechas de género son más profundas entre los indígenas rurales: en 2002 el porcentaje de hombres con 4 años o menos de escolaridad era entre 6 y 13 p.p. menos que las mujeres dependiendo de la región. En 2017 ya no había diferencia de género en la región Central, mientras que en el Sur y Norte subsistía una ventaja de 5 y 7 p.p. respectivamente.

En conclusión, en Colombia, Chile y Perú las mayores brechas de género se dan entre los indígenas de la zona rural. Pero hay bastante variación entre los países: en Chile y Colombia las diferencias entre hombres y mujeres son moderadas, mientras que en Perú llegan a ser muy profundas. En Perú también se dan importantes brechas de género en categorías como indígenas urbanos y no indígenas rurales, categorías en las que este tipo de brechas ya se han superado en los otros países analizados.

\section{Las intersecciones con enfoque de ruralidad}

La brecha entre la región urbana y rural es profunda y constante en los países de la región. No obstante, es importante señalar que algunas intersecciones agravan y otras atenúan las brechas entre el área urbana y rural. En Colombia la brecha pasó de 14 a 8 p.p. entre las dos rondas censales; en Perú pasó de 15 a 14 p.p. mostrando una persistencia de la desigualdad; mientras que en Chile la brecha de escolaridad se redujo considerablemente, de 20 a 10 p.p.

En Colombia la brecha urbano-rural entre los no identificados con ninguna etnia fluctuaba entre 4 y 18 p.p. en 2005 y entre 4 y 13 p.p. en 2018 dependiendo de la región. La región Amazonía-Orinoquía era la que más atenuaba la brecha y la región Caribe era la que más la agravaba, especialmente para los hombres.

Las brechas urbano-rurales para los afrodescendientes son similares que para los no identificados con ninguna etnia. Pero la brecha urbano-rural se profundiza sustancialmente entre los indígenas. La diferencia en la tasa de analfabetismo entre los indígenas urbanos y los rurales oscila entre 9 y 33 p.p. para hombres y 12 y 38 p.p. para mujeres en la primera ronda censal y dependiendo de la región. Trece años después, en 2018, la diferencia urbano-rural se redujo considerablemente, pero sigue siendo alta: entre 2 y 19 p.p. para hombres y 5 y 21 p.p. para mujeres. Las regiones Caribe y Oriental son las que profundizan más la brecha urbano-rural entre la población indígena y las regiones Pacífica y Amazonía-Orinoquía son las que más la atenúan.

En Perú no importa si se está en la Costa, la Selva o la Sierra, la brecha urbano-rural está muy mediada por el género y la etnia. Ser hombre atenúa mucho la penalidad de la ruralidad: entre los hombres que aprendieron castellano como primera lengua la brecha entre la tasa urbana (1\%) y la rural $(6 \%-9 \%)$ es de 5 a 8 p.p. dependiendo de la región y se mantiene en 2017. Entre los hombres que aprendieron lenguas indígenas la brecha entre la tasa urbana (3-6\% según región) y la rural (10-15\% según región) es de aproximadamente 8 p.p. y varía 
muy poco entre regiones. Vemos así que ser hombre, incluso hombre indígena, protege bastante de la condición de analfabetismo en la ruralidad.

En cambio, ser mujer agrava tremendamente la penalidad de la ruralidad. Entre las castellano-hablantes la brecha en 2007 fluctuaba entre 12 y 20 p.p. dependiendo de la región y entre 12 y 18 p.p. en 2017, una brecha 10 p.p. más profunda que en los hombres. Vemos así que no solo la brecha prácticamente no cambia en diez años, sino que para las mujeres rurales en Perú la región sí importa, pues la brecha urbano-rural es menor en la Selva que en las otras dos. Pasando a la brecha urbano-rural entre mujeres indígenas ésta fluctúa entre 15 p.p. y 25 p.p. en 2007, reduciéndose a entre 12 y 15 p.p. en 2017 según región. Si bien había una diferencia regional importante en 2007 esta se ha recortado bastante en 2017.

En Chile la brecha urbano-rural es importante y en algunas categorías presenta variación regional. La brecha urbano-rural entre las personas que no hacen parte de ninguna etnia era entre 14 y 20 p.p. en 2002 y 6 y 10 p.p. en 2017 y no hay importantes diferencias de género. Entre los dos años evaluados se ha recortado bastante la brecha y la fluctuación regional.

Entre los indígenas la brecha urbano-rural era más grande y ha tenido una reducción muy sustancial. Entre las mujeres la brecha era un poco más profunda, pues la diferencia en la tasa de personas con cuatro años o menos de escolaridad entre la ciudad y el campo era entre 20 y 25 p.p. en 2002 y 6 y 10 p.p. en 2017. Entre los hombres la brecha y la variación regional era menor: entre 18 y 21 p.p. en 2002 y entre 5 y 12 p.p. en 2017. Vemos así como la condición de ruralidad limita las posibilidades de acceder a la educación, incluso más si uno es indígena, mujer y se encuentra en la región Sur.

En conclusión, en los tres países las oportunidades de educación son muy distintas para el campo y la ciudad. La penalidad de la ruralidad es bastante menor para los que no se identifican con ninguna etnia en Colombia, mientras que en Chile la penalidad de la ruralidad es parecida para los indígenas y no indígenas, y en Perú esta depende del género. De hecho, en Chile las brecha urbano-rural se profundiza un poco para las mujeres, mientras que en Perú la brecha se profundiza dramáticamente. En Colombia la brecha urbano-rural es igual para hombres y mujeres. Finalmente, La región no genera mucha variación en las brechas urbano rurales de Perú, excepto para mujeres, mientras que en Chile hay una variación un poco más significativa y en Colombia hay una variación regional mayor en las brechas urbano-rurales.

\section{Las intersecciones con enfoque étnico}

La profundidad de la brecha entre grupos étnicos varía entre países. En Colombia la diferencia en la tasa de analfabetismo entre los que no se identificaban con ninguna etnia y los afrodescendientes era de 5 p.p. en 2005 y 3 p.p. en 2018. La brecha se profundiza con los indígenas: del 23 p.p. en 2005 y 13 p.p. en 2018. 
En Perú la brecha entre los indígenas y los no indígenas pasó del 17 p.p. a 13 p.p. entre 2007 y 2017; mientras que en Chile la brecha pasó de 11 p.p. a 3 p.p.

En Colombia la brecha étnica (entre indígenas y no identificados con ninguna etnia) es significativa en el área rural y aún más entre las mujeres: la diferencia en tasa de analfabetismo para hombres rurales indígenas y no indígenas fluctúa entre 5 y 23 p.p. para 2005 y 2 y 10 p.p. para 2018 según región, mientras que para mujeres la brecha étnica va entre 14 y 38 p.p. en 2005 y 7 y 18 p.p. en 2018 según región. Vemos que en el área rural, y sobre todo entre las mujeres, la variación regional es importante. La brecha étnica se siente mucho menos en la Amazonía-Orinoquía y la región Pacífica, y mucho más en la región Caribe y Oriental. Llama mucho la atención la región Caribe, pues la diferencia en la tasa de analfabetismo entre las indígenas rurales y las no indígenas rurales era de 34 p.p. en 2005 y 18 p.p. en 2018. En conclusión, en Colombia la brecha étnica se atenúa en el área urbana, sobre todo entre los hombres, y se profundiza en el área rural, y se agrava críticamente en la región Caribe.

En Perú la brecha étnica es prácticamente invisible entre los hombres urbanos, pues la diferencia en tasa de analfabetismo entre los que aprendieron lenguas indígenas y español es del 2 a 4 p.p. en ambas rondas censales. Entre las mujeres urbanas, la brecha étnica es mucho más visible, pues varía entre 14 p.p. y 20 p.p. para 2007 y entre 11 p.p. y 16 p.p. en 2017. La mayor brecha étnica se encuentra en la Sierra.

La brecha étnica en hombres rurales es de 5 p.p. en 2017 y 7-9 p.p. en 2007, excepto en la Costa en donde no hay brecha étnica dentro de lo rural. Ésta, como hemos dicho en los anteriores análisis, se profundiza entre las mujeres, que sufren la misma penalidad en todas partes: entre 18 p.p. y 23 p.p. en 2007 y entre 14 y 16 p.p. en 2017. La región hace poco para la brecha étnica en Perú.

En Chile la brecha étnica es muy pequeña entre los hombres urbanos, y un poco mayor entre las mujeres urbanas, y ambas se han reducido a casi su totalidad según el último censo. En el área rural se siente más la brecha étnica especialmente entre las mujeres (10 a 13 p.p. para 2002 y 3 a 8 p.p. para 2017), y en los hombres pero sólo de la región Sur. Entre los dos censos la diferencia entre etnias se ha reducido, aunque subsiste entre las mujeres de la región sur.

En conclusión, la brecha étnica se agrava en la zona rural, especialmente en las mujeres, aunque en Perú se observan importantes diferencias entre etnias entre mujeres de la zona urbana también. En Perú, a pesar de la severidad de la brecha étnica, hay poca variación regional, mientras que en Colombia ésta es bastante amplia. En Chile subsisten las brechas étnicas solo entre las mujeres rurales.

\section{Las intersecciones con enfoque regional}

Para finalizar el análisis volcamos la mirada a las diferencias que hace vivir en una región u otra. En el agregado, la brecha regional es una de las más pe- 
queñas y es parecida entre los países. En Colombia la diferencia entre la región con mayor y menor analfabetismo era de $8 \%$ en 2005 y 5\% en 2018. En Perú la diferencia alcanza a ser de 9 p.p. en 2007 y 7 p.p. en 2017. Chile la diferencia entre la región más y menos afectada por la falta de escolaridad era de 9 p.p. en 2002 y 4 p.p. en 2017.

Cuando introducimos las otras características en el análisis encontramos una gran variación en la variación regional. En Colombia hay algunas intersecciones con muy poca variación regional, y otras con una variación enorme. Entre las primeras, la mayor diferencia entre regiones para las mujeres y los hombres urbanos no identificados con ninguna etnia es de 4 p.p., y se ha recortado a 2 p.p. en 2018. Entre los urbanos que se identifican como indígenas la brecha regional aumenta un poco, 12 p.p. para 2005 y 5 p.p. para 2017 entre los hombres, aunque vale anotar que la brecha regional para las mujeres urbanas indígenas es menor. Vemos así que ser de una región u otra no es una condición suficiente para la desventaja: para algunas categorías la región no hace diferencia en las posibilidades de saber leer y escribir.

Naturalmente, la brecha regional se intensifica en el área rural: 13 p.p. en 2005 y 9 p.p. en 2018 entre los hombres no indígenas y 10 p.p. en 2005 y 6 p.p. entre las mujeres no indígenas. Para los indígenas rurales la brecha regional se agudiza y es similar en hombres y mujeres: casi 30 p.p. es la diferencia entre la región con más y menos tasa de analfabetismo en 2005, y 20 p.p. en 2018. Esta variación enorme sugiere que hay unas regiones donde ser indígena limita enormemente las posibilidades de acceder a la educación básica.

En Perú, como lo hemos mencionado anteriormente, la variación regional es mucho más atenuada. En hombres urbanos -tanto indígenas como no indígenas - la brecha regional es de máximo 3 p.p., al igual que para mujeres urbanas no indígenas, hombres rurales no indígenas, y curiosamente, mujeres rurales indígenas. La brecha regional se siente solo en hombres rurales indígenas (5 p.p. y 3 p.p. entre los dos años), mujeres urbanas indígenas (8 p.p. y 5 p.p. entre los dos años), y mujeres rurales no indígenas (7 p.p. y 3 p.p.). La brecha regional es mucho menor que en Colombia y se ha disminuido bastante para 2017.

En Chile la brecha regional pesaba un poco más que en Perú en el año 2002: en casi todos los grupos la brecha regional estaba entre 3 p.p. y 6 p.p. En apenas dos grupos la región ejercía más diferencias: entre los hombres y las mujeres rurales indígenas, con brechas regionales de 8 y 10 p.p. En 2017 la brecha regional se recortó a 2-3 p.p. en casi todos los grupos. Los grupos en los que subsisten diferencias entre regiones son las mujeres rurales indígenas (13 p.p. de diferencia entre la región Central y la Sur) y los hombres rurales indígenas (8 p.p. de diferencia entre las mismas regiones).

En conclusión, si bien los tres países tienen brechas regionales similares en el agregado, el análisis desagregado por intersecciones de categorías refleja unas tendencias muy distintas. En Perú hay muy poca variación regional, incluso 
en las categorías en mayor desventaja, y la brecha se ha reducido a una mínima expresión en 2017. En Chile la brecha regional también se recortó en todas las intersecciones, excepto en los indígenas rurales. En Colombia, a diferencia de los otros dos países, subsisten importantes brechas en la ruralidad, incluso entre personas que no pertenecen a grupos étnicos. Pero las brechas regionales entre indígenas rurales son extraordinarias, pues la diferencia entre la región con más y menos tasa de analfabetismo llega a ser 20 p.p.

\section{COMPRENDIENDO EL ROL DE LA REGIÓN COMO DETERMINANTE DE DESIGUALDAD}

Para entender mejor la naturaleza de la brecha regional, o desigualdad subnacional, esta sección presenta los resultados de la descomposición Oaxaca-Blinder, cuya metodología se describió en la sección III. Escogí para cada país las dos regiones con mayores contrastes en los niveles de desarrollo social: Caribe y Andina Central para Colombia, Sierra y Costa para Perú y Central y Sur para Chile. Las brechas entre estas regiones son frecuentemente analizadas en la literatura regional y las oficinas de planeación y estadística. Si bien este análisis podría repetirse para otras regiones, no tengo el espacio suficiente para ello.

El análisis permite identificar qué parte de la diferencia entre las regiones en las tasas de analfabetismo y escolaridad corresponden a la distribución de jóvenes, campesinos, mujeres y miembros de minorías étnicas. Esto, que la literatura llama porción explicada, remite a un componente composicional en la desigualdad subnacional. Es decir, a la parte de la brecha que responde a distintas composiciones de los grupos sociales que se encuentran en cada región.

El resto de la brecha se conoce como la porción no explicada. Como se recordará, parte de esta porción no explicada se explica por diferencias en los efectos (o los coeficientes) que tiene ser mujer, ser campesino o ser minoría indígena en las regiones desventajadas. Es decir, tiene que ver con la forma como dentro de la región interactúan de forma particular los atributos de género, ruralidad y etnia. Por ello la porción no explicada se refiere en parte a un componente interseccional de la brecha.

Se utilizó el módulo de stata Fairlie (Jann 2008) que adapta el método Oaxaca-Blinder para variables binarias con regresiones no lineales (Fairlie 2006). Los resultados se presentan a continuación.

La descomposición ratifica la idea que la naturaleza de la desigualdad subnacional es muy distinta entre países y que cambia en el tiempo, a pesar que en términos absolutos la magnitud de la brecha entre la región con más analfabetismo/escolaridad y menos analfabetismo/escolaridad es parecida. Como vemos en la tabla 2, la diferencia entre la región andina y la región Caribe en Colombia en la tasa de analfabetismo era el 8\% en 2005 y 5\% en 2018. En Perú, la diferencia en la tasa de analfabetismo entre la Costa y la Sierra era de $9 \%$ en 
2007 y $7 \%$ en 2017. En Chile, la diferencia en escolaridad entre la región Central y la Sur era de 7\% en 2002 y 3\% en 2017.

Tabla 2. Resultado de la descomposición Oaxaca-Blinder para Colombia, Perú y Chile.

\begin{tabular}{|c|c|c|c|c|c|c|c|}
\hline & & \multicolumn{2}{|c|}{ Colombia } & \multicolumn{2}{|c|}{ Perú } & \multicolumn{2}{|c|}{ Chile } \\
\hline & & 2005 & 2018 & 2007 & 2017 & 2002 & 2017 \\
\hline \multicolumn{2}{|l|}{ Región 1} & \multicolumn{2}{|c|}{ Andina } & \multicolumn{2}{|c|}{ Costa } & \multicolumn{2}{|c|}{ Central } \\
\hline \multicolumn{2}{|l|}{ Región 2} & \multicolumn{2}{|c|}{ Caribe } & \multicolumn{2}{|c|}{ Sierra } & \multicolumn{2}{|c|}{ Sur } \\
\hline \multicolumn{2}{|c|}{ Tasa de Analfabetismo Región 1} & $8 \%$ & $5 \%$ & $4 \%$ & $3 \%$ & $13 \%$ & $10 \%$ \\
\hline \multicolumn{2}{|c|}{ Tasa de Analfabetismo Región 2} & $15 \%$ & $10 \%$ & $13 \%$ & $10 \%$ & $20 \%$ & $13 \%$ \\
\hline \multicolumn{2}{|c|}{ Diferencia (Región 1- Región 2) } & $8 \%$ & $5 \%$ & $9 \%$ & $7 \%$ & $7 \%$ & $3 \%$ \\
\hline \multirow{5}{*}{$\begin{array}{l}\% \text { explicado } \\
\text { por diferencias } \\
\text { composicionales entre } \\
\text { las regiones }\end{array}$} & & $16 \%$ & $17 \%$ & $86 \%$ & $91 \%$ & $63 \%$ & $37 \%$ \\
\hline & Etnias & $87 \%$ & $111 \%$ & $42 \%$ & $45 \%$ & $23 \%$ & $18 \%$ \\
\hline & Urbano-Rural & $82 \%$ & $98 \%$ & $75 \%$ & $73 \%$ & $85 \%$ & $79 \%$ \\
\hline & Edad & $-69 \%$ & $-109 \%$ & $-11 \%$ & $-10 \%$ & $-7 \%$ & $3 \%$ \\
\hline & Género & $0 \%$ & $0 \%$ & $-6 \%$ & $-9 \%$ & $-1 \%$ & $0 \%$ \\
\hline
\end{tabular}

Nota: todos los porcentajes son significativos a 95\% IC excepto género en Colombia 2005.

Ahora identifiquemos la porción explicada y no explicada de esa brecha. En Colombia encontramos que apenas el 16\% (2005) y 17\% (2018) de la diferencia entre las regiones es explicada, o se da por las diferencias en la composición de las regiones. En Perú casi toda la diferencia entre la Costa y la Sierra es explicada por las diferencias composicionales, y Chile está entre los dos países: en 2002 el 63\% de la diferencia entre la región Central y la Sur era explicada por diferencias composicionales, pero en 2017 esa proporción se redujo a solo 37\%.

¿Cómo interpretamos esos resultados? La descomposición sugiere que en Colombia las diferencias observadas entre las regiones no se deben en gran medida (solo 16-17\%) a las diferencias en la proporción de jóvenes, indígenas, afrodescendientes, mujeres y pobladores rurales que habitan en las regiones. En otras palabras, si la región Caribe tuviera la misma proporción de minorías étnicas, mujeres y pobladores rurales que la Andina Central, la diferencia que observamos entre las dos regiones en la tasa de analfabetismo se reduciría solo en $16 \%$ o $17 \%$, que equivale a solo 1 p.p. De ese 16 o $17 \%$ que es explicado, casi toda la diferencia la hace la composición étnica y la composición urbana/rural. Por otro lado, el otro $84 \%$ no explicado de la diferencia entre la región Caribe y la Andina se debe en parte a que cada una de las categorías incluidas tiene efectos distintos en las regiones: por ejemplo, es probable que ser indígena disminuya más las probabilidades de acceder a educación en la región caribe que en la andina. La importancia de la porción no explicada da evidencia a favor del enfoque interseccional, puesto que estas categorías interactúan con la región para producir distintas oportunidades y obstáculos en el acceso a competencias educativas básicas. 
La situación es distinta en Perú. En este país el 86\% (2007) y 91\% (2017) de la diferencia en las tasas de analfabetismo entre la Costa y la Sierra se deben a diferencias composicionales. En otras palabras, si la Sierra tuviera la misma proporción de jóvenes, habitantes urbanos, mestizos y hombres que la Costa, su tasa de analfabetismo sería casi igual. El mayor responsable de la diferencia entre las regiones es la composición urbano-rural y en segunda medida la étnica. En Perú no parece que la región interactúe con las características de las personas para producir efectos diferenciados en el acceso a la educación más básica.

Finalmente, Chile está entre ambos mundos. En 2002 el 63\% de la diferencia en la proporción de personas con cuatro o menos años de escolaridad era explicado por diferencias composicionales. El efecto más importante lo tiene la composición urbano-rural, pues de ser igual en ambas regiones la brecha se reduciría en 4 p.p. Pero en 2017 se reduce la porción explicada y aumenta la porción no explicada. El 37\% de la pequeña brecha de 3 p.p. se explica por la distinta composición, sobre todo en lo que tiene que ver con lo urbano-rural. El 63\% de la brecha no está explicada. Es altamente probable que parte de esta porción no explicada se deba a que en la región Sur ser indígena o campesino tenga una mayor penalidad sobre la escolaridad que en la región Central, - aunque el efecto es pequeño puesto que la brecha es muy reducida.

Los hallazgos obtenidos con la descomposición Oaxaca-Blinder están alineados con los de la sección anterior. El hecho de que sea Colombia el país con mayor proporción de la brecha no explicada, seguido por Chile y Perú nos remite a lo observado en cuando a que las brechas étnicas y urbano-rurales y de género tenían mucha más variación regional en Colombia, seguido por Chile, y menor en Perú. El significado de estas coincidencias se explorará a continuación.

\section{La naturaleza de la desigualdad subnacional}

La descomposición Oaxaca-Blinder nos invita a reflexionar sobre la naturaleza de la desigualdad subnacional. ¿Existe una fuerza causal específica de la región que produce privilegios y opresiones, y que -aunque interactúe con- sea independiente del racismo, el sexismo y el sesgo urbano? ¿O, por el contrario, la región no tiene una fuerza causal propia, sino que es un espacio donde simplemente se expresan las discriminaciones existentes y la magnitud de la brecha es función del tamaño de las minorías, los campesinos y las mujeres? La alta porción explicada en la descomposición nos invitaría a pensar lo segundo. No obstante, mi invitación es que incluso cuando casi toda la desigualdad subnacional se explica por la composición de las regiones, existe una fuerza causal regional en acción. Esto es porque no es casualidad que la región tenga dicha composición.

La descomposición realizada en la sección anterior nos permite entender la desigualdad subnacional -o la brecha entre regiones- como la conjunción de 
dos tipos de fenómenos. Por un lado, parte de la brecha se debe a diferencias en la composición de las regiones. Unas regiones tienen más pobladores rurales, y más minorías étnicas por distintas razones aleatorias económicas, históricas, políticas y ecológicas y eso produce desventajas y privilegios. Por dicha composición en las regiones se reflejan dinámicas de racismo, el sexismo, y el sesgo rural $^{6}$, que son comunes en el conjunto de la nación pero que se expresan con más fuerza en ciertas regiones en función de su número de minorías.

Otra parte de la brecha o de la desigualdad regional responde a la forma como la interacción particular entre etnia, la condición urbana-rural, el género, y otras características, producen efectos diferenciados en cada región. En otras palabras, parte de la desigualdad regional se da porque unas regiones acentúan el sexismo, el racismo y el sesgo rural y sus interacciones. En otras literaturas se le conoce a esto como "efectos heterogéneos", "retornos", o "susceptibilidades" (Jackson y VanderWeele 2019). Mi propuesta es llamar a estas dos porciones de desigualdad subnacional como la composicional y la interseccional.

La descomposición anterior permite discriminar entre ambos tipos. Como vimos, los componentes de la brecha varían de país en país y en el tiempo. En Chile y Perú el componente composicional es más importante, mientras que en Colombia es el menos importante. Pero en vez de asumir que un elevado componente composicional significa que no existe una fuerza causal propia de la región, nos debemos preguntar por qué las regiones con mayores (y menores) desventajas tienen esas composiciones. Esto se da no por razones accidentales, sino por distintas trayectorias históricas y configuraciones de economía política.

Un ejemplo claro es el de las causas del rezago de la región caribe en Colombia. Esta parte del país tiene mayor proporción de habitantes rurales no por casualidad, sino porque las élites políticas que fundaron la república pusieron en marcha un proyecto ideológico de construcción de nación según el cual Colombia era un país de regiones, pero donde la región andina debía ser dominante frente a las demás debido a su composición racial más blanca (Múnera 2005). El modelo económico de sustitución de importaciones en Colombia, desarrollado desde los años 50s, reforzó el privilegio de la región andina y allí concentró el desarrollo industrial y el crecimiento económico, en parte porque allí se acumulaban los excedentes de la producción cafetera. Esto conllevó a mayores inversiones en educación pública, salud y vivienda en esta región, así como una mejor infraestructura para la productividad, expresada en, por ejemplo, una energía basada en hidroeléctricas, más barata, más confiable, y más eficiente que la energía térmica de la región caribe. Las diferencias composicionales no son entonces causadas por elecciones individuales de los habitantes del caribe, que deciden o no migrar y dedicarse a otras actividades, sino por proyectos 
ideológicos excluyentes y economía política que limitaron allí su desarrollo (Meisel 1999, Meisel y Hahn 2020).

El ejemplo de la región Caribe ilumina el hecho que la región como espacio no es una tabula rasa donde se expresan racismos, sexismos y discriminaciones en función del número de minorías. Al contrario, son los procesos políticos, económicos e históricos enraizados en el espacio de la región, articulados por élites regionales, y avanzados por instituciones subnacionales lo que explica su particular composición, que a su vez explica parte de la desigualdad subnacional.

De igual forma en Perú la alta proporción de habitantes rurales e indígenas en la región Sierra no solo se debe a los patrones de poblamiento precolonial, sino a la emergencia de la Costa como centro de dominación tras la independencia. La predominancia de la costa se selló con el boom del guano y la devastación de la sierra en la guerra del pacífico, y después con la promoción de exportaciones de petróleo, pescado, azúcar y algodón, todas centradas en la Costa. Instituciones políticas tales como la exigencia de alfabetismo para votar excluyeron de tajo la participación de los grupos indígenas y la representación de sus intereses, profundizando la marginación de los territorios por ellos habitados. Capas de dinámicas políticas y económicas fueron creando composiciones muy distintas de grupos sociales privilegiados y marginados en cada una de las regiones (Thorp y Paredes 2010).

La dimensión subnacional como una categoría o un atributo con una fuerza causal propia que produce privilegios y marginaciones se hace un tanto más evidente en el componente interseccional. Dicha fuerza causal se refleja en que las categorías sociales como la raza y el género interactúan entre ellas y con la región para producir efectos heterogéneos, parte de lo cual es capturado por la porción "no explicada" de la descomposición. La evidencia de la existencia de esta desigualdad conlleva a otro tipo de preguntas: ¿Por qué ser mujer e indígena tiene un impacto distinto en las oportunidades de acceder a la educación en distintas regiones? ¿Por qué les va peor a los hombres campesinos en unas regiones que en otras? La respuesta a estas preguntas necesariamente apunta al rol de las élites regionales, a la formación local del estado, a prácticas culturales específicas y a las acciones de líderes de las comunidades campesinas e indígenas. Puede ser que las iniciativas educación intercultural que pretenden adaptarse a las necesidades de los grupos indígenas se hayan ejecutado mejor en unas regiones que en otras. Puede ser también que grupos indígenas específicos ubicados en ciertos espacios tengan unas concepciones de la educación de las mujeres más limitantes que otros. También es posible que las élites políticas de la región más desventajada hayan desfinanciado sistemáticamente a las zonas con minorías para limitar las demandas de población oprimida, algo que no ocurrió en tal magnitud en las otras regiones. O que existan diferencias culturales marcadas en la forma como se concibe el lugar de la mujer, lo que se traduce en una menor infraestructura para la educación y el trabajo de las mujeres en unas regiones que en otras. $\mathrm{O}$ a lo mejor responde a la existencia de vínculos clientelistas entre las élites y los grupos marginados, que por un lado 
facilitan la reproducción de dichas elites en el poder y por otro reproduce mantiene las condiciones estructurales de pobreza. Todas estas dinámicas apuntan a interacciones específicas entre género, etnia, ruralidad con las dinámicas políticas o económicas subyacentes a regiones específicas.

De este modo, distinguir entre el componente composicional y el interseccional no solo ayuda a identificar cuáles son las políticas públicas apropiadas para cerrar la brecha, sino que también ayuda a identificar los agentes que la producen y reproducen. Tanto agentes y procesos dentro y fuera de la región, actuales e históricos, confluyen en crear la desigualdad regional. Esta conceptualización de la desigualdad subnacional evidencia que para explicarla es necesario acudir a teorías multinivel que tengan en cuenta agentes y procesos en distintas escalas (Giraudy et al. 2019).

\section{CONCLUSIONES}

La investigación subnacional en América Latina ha otorgado suficiente evidencia sobre cómo la calidad de vida, la provisión de bienes públicos y las dinámicas de desarrollo varían de región en región. Pero sabemos aún muy poco de por qué se da esta variación: si es porque la región es un simple reflejo de marginaciones y discriminaciones de género, raza y etnia, o si de hecho lo subnacional tiene una fuerza causal independiente.

Este artículo responde a esta pregunta desde el enfoque interseccional y sus metodologías cuantitativas. Así, partimos de comprender lo subnacional como un atributo o categoría social que interactúa con el género, la etnia y la condición de ruralidad para producir variaciones en marginaciones y privilegios. A partir del análisis de la distribución en la tasa de analfabetismo o escolaridad en grupos compuestos por la intersección de estos atributos, concluimos que las brechas étnicas, de género, y urbano-rurales se agravan y atenúan en unas regiones, y que a veces estas brechas tienen importantes variaciones regionales, $\mathrm{y}$ otras veces no.

La descomposición Oaxaca-Blinder permitió avanzar en la comprensión de la naturaleza de dichas brechas regionales y sus diferencias. Se puede teorizar que parte de las brechas regionales tienen una naturaleza composicional y otra parte tiene una naturaleza interseccional y el peso de cada una varía de país en país y a lo largo del tiempo. Para ambos componentes existe una fuerza causal subyacente a lo subnacional que -aunque es independiente- actúa en interacción con la raza, el sexo, y el género en la producción de desigualdades.

Es así como procesos históricos, económicos y políticos han privilegiado el desarrollo de unas regiones sobre otras, afectando sus composiciones, y también albergando modos diferenciados de interacción entre género, étnica y clase que se destilan en prácticas culturales, trayectorias educativas, articulaciones políticas e instituciones concretas. La comprensión de la desigualdad subna- 
cional desde las perspectivas composicionales e interseccionales nos permite ganar mucha complejidad en la comprensión del problema. No se trata así solo de promover migración del campo a la ciudad o políticas de asimilación de gurpos indígenas en las regiones desventajadas. Se trata de comprender cómo instituciones políticas, prácticas culturales y proyectos hegemónicos que están anclados en espacios concretos promueven que interacciones de raza-género y clase sean particularmente discriminatorias.

Esta investigación pretende contribuir a profundizar la comprensión de la naturaleza de las brechas regionales o subnacionales y motivar futuras investigaciones en este campo. Hacerlo permitirá identificar políticas públicas contextualmente acertadas e intervenciones que visibilicen la experiencia de grupos sociales en la intersección de varios atributos tradicionalmente marginados. El avance hacia una mayor igualdad de oportunidades requiere estas lecturas que trasciendan la tiranía de los promedios.

\section{BIBLIOGRAFÍA}

Abramo, Laís. 2019. "La Matriz de la Desigualdad en América Latina.: Avances Y Desafíos de Cara Al Futuro". En Las Sendas Abiertas en América Latina, Aprendizajes Y Desafíos Para Una Nueva Agenda de Transformaciones, editado por Daniel Filmus y Lucila Rosso. CLACSO, 99-136.

Antía, Florencia. 2018. “Regímenes de Política Social en América Latina: Una Revisión Crítica de la Literatura. Desafíos 30(2): 193-235

Babb, Florence E. 2017. “Desigualdades Entrelazadas: Repensando la Raza, El Género Y Las Identidades Indígenas en El Perú Andino". En Racismo y Lenguaje, editado por Virginia Zavala y Michele Back, Lima: Pontificia Universidad Católica de Perú: 229-268.

Baquero Melo, Jairo. 2017. “Desigualdades Superpuestas, Capas de Desigualdad e Interseccionalidad: Consideraciones Analíticas y Aplicación al Caso Colombiano". Análisis Político 30(89): 59-75.

Bauer, Greta R. 2014. "Incorporating Intersectionality Theory into Population Health Research Methodology: Challenges and the Potential to Advance Health Equity". Social Science \& Medicine 110: 10-17.

Bauer, Greta R., y Ayden I. Scheim. 2019. "Methods for Analytic Intercategorical Intersectionality in Quantitative Research: Discrimination as a Mediator of Health Inequalities." Social Science \& Medicine 226: 236-245.

Beramendi, Pablo. 2012. The Political Geography of Inequality: Regions and Redistribution. New York: Cambridge University Press.

Bértola, Luís. 2020. "Productive and Regional Development Policies in Latin America Since 1890". En Time and Space: Latin American Regional Development in Historical Perspective, editado por Daniel A. Tirado-Fabregat, Marc Badia-Miró, y Henry Willebald. Cham: Springer International Publishing, 41-64.

Bértola, Luis, y Jeffrey Williamson. 2017. Has Latin American Inequality Changed Direction?: Looking Over the Long Run. Cham: Springer.

Blinder, Alan S. 1973. "Wage Discrimination: Reduced Form y Structural Estimates." Journal of Human Resources 8(4): 436-455.

Boesten, Jelke. 2010. Intersecting Inequalities: Women and Social Policy in Peru, 1990-2000. Pennsylvania: Penn State Press. 
Bowleg, Lisa. 2012. “The Problem With the Phrase Women y Minorities: Intersectionality—an Important Theoretical Framework for Public Health." American Journal of Public Health 102 (7): 1267-73.

Bowleg, Lisa, y Greta Bauer. 2016. "Invited Reflection: Quantifying Intersectionality". Psychology of Women Quarterly 40(3): 337-41.

Browne, Irene, y Joya Misra. 2003. "The Intersection of Gender y Race in the Labor Market." Annual Review of Sociology 29(1): 487-513.

Busso, Matías, y Julián Messina, eds. 2020. La Crisis de la Desigualdad. América Latina y el Caribe en la Encrucijada. Washington D.C: Banco Interamericano de Desarrollo.

Caro Molina, Pamela. 2017. “Desigualdad y Transgresión en Mujeres Rurales Chilenas: Lecturas Desde la Interseccionalidad, Género y Feminismo". Psicoperspectivas 16(2): 125-37.

Collins, Patricia Hill. 2015. "Intersectionality's Definitional Dilemmas". Annual Review of Sociology 41(1): 1-20.

Crenshaw, Kimberlé. 1991. "Mapping the Margins: Identity Politics, Intersectionality, and Violence against Women." Stanford Law Review 43(6): 1241-1299.

Dell, Melissa. 2010. "The Persistent Effects of Peru's Mining Mita". Econometrica 78(6): 18631903.

Else-Quest, Nicole M., y Janet Shibley Hyde. 2016. "Intersectionality in Quantitative Psychological Research: I. Theoretical and Epistemological Issues". Psychology of Women Quarterly 40(2): 155-170.

Evans, Clare R. 2019. "Modeling the Intersectionality of Processes in the Social Production of Health Inequalities." Social Science \& Medicine 226: 249-53.

Evans, Clare R., David R. Williams, Jukka-Pekka Onnela, y S. V. Subramanian. 2018. "A Multilevel Approach to Modeling Health Inequalities at the Intersection of Multiple Social Identities". Social Science \& Medicine 203: 64-73.

Fairlie, Robert W. 2006. An Extension of the Blinder-Oaxaca Decomposition Technique to Logit and Probit Models. Rochester, NY: Social Science Research Network. SSRN Scholarly Paper. Recuperado el 15 de julio de 2020 de https: / / papers.ssrn.com/abstract $=497302$

Giraudy, Agustina, Eduardo Moncada, y Richard Snyder, eds. 2019. Inside Countries: Subnational Research in Comparative Politics. New York: Cambridge University Press.

Giraudy, Agustina, y Jennifer Pribble. 2020. “Territorial Inequality in Health Service Delivery: Lessons from Latin America's Federations". Latin American Politics and Society 62(3): 19-43.

Gopaldas, Ahir. 2013. "Intersectionality 101". Journal of Public Policy \& Marketing 32(1_suppl): 90-94.

Hancock, Ange-Marie. 2007. “When Multiplication Doesn't Equal Quick Addition: Examining Intersectionality as a Research Paradigm." Perspectives on Politics 5(1): 63-79.

Harbers, Imke. y Abbey Steele. 2020. "Subnational Variation Across States: A Typology and Research Agenda". Latin American Politics and Society 62(3):1-18.

Hlavac, Marek. 2014. “Oaxaca: Blinder-Oaxaca Decomposition in R.” SSRN Scholarly Paper ID 2528391. Rochester, NY: Social Science Research Network.

Hoffman, Kelly, y Miguel Angel Centeno. 2003. “The Lopsided Continent: Inequality in Latin America." Annual Review of Sociology 29 (1): 363-90.

Hopkins, Peter. 2019. "Social Geography I: Intersectionality." Progress in Human Geography 43(5): 937-47.

Jackson, John W. y Tyler J. VanderWeele. 2019. “Intersectional Decomposition Analysis with Differential Exposure, Effects, and Construct". Social Science \& Medicine 226: 254-59.

Jann, Ben. 2008. FAIRLIE: Stata Module to Generate Nonlinear Decomposition of Binary Outcome Differentials. Recuperado el 15 de julio de 2020 de https:/ /econpapers.repec.org/software/bocbocode/s456727.htm

Kabeer, Naila, y Ricardo Santos. 2017. Intersecting Inequalities and the Sustainable Development Goals: Insights from Brazil. WIDER Working Paper Series 167. Helsinki: World Institute for Development Economic Research (UNU-WIDER). 
Krizsan, Andrea, Hege Skjeie, y Judith Squires. 2012. Institutionalizing Intersectionality: The Changing Nature of European Equality Regimes. Cham: Springer.

Krugman, Paul R. 1991. Geography and trade. Boston: MIT press.

López-Calva, Luis Felipe, y Nora Claudia Lustig. 2010. Declining Inequality in Latin America: A Decade of Progress? Washington D.C: Brookings Institution Press.

Lustig, Nora. 2020. "Desigualdad y Descontento Social en América Latina". Nueva Sociedad (286): 53-61.

Lustig, Nora, y Jorge Mariscal. 2020. “El Impacto de la Covid-19 en América Latina: Se Requieren Respuestas Fuera del Libreto". Análisis Carolina 22/2020

Malmusi, Davide, Carme Borrell, y Joan Benach. 2010. "Migration-Related Health Inequalities: Showing the Complex Interactions between Gender, Social Class y Place of Origin." Social Science \& Medicine 71(9): 1610-19.

Martínez, Rodrigo, Daniela Trucco, y Amalia Palma. 2014. "El Analfabetismo Funcional en América Latina y el Caribe: Panorama y Principales Desafíos de Política". Serie Políticas Sociales No. 198.

Meisel Roca, Adolfo. 1999. “¿Por Qué Perdió la Costa Caribe el siglo XX?” Revista del Banco de la República 72: 19-47.

Meisel Roca, Adolfo y Lucas Hahn. 2020. "Regional Economic Inequality in Colombia, 19262018". En Time and Space: Latin American Regional Development in Historical Perspective, editado por Daniel A. Tirado-Fabregat, Marc Badia-Miró, y Henry Willebald. Cham: Springer International Publishing, 183-210.

Minnesota Population Center. 2019. Integrated Public Use Microdata Series, International: Version 7.2 [base de datos]. Minneapolis: IPUMS.

Molinas Vega, José R., Ricardo Paes de Barros, Jaime Saavedra Chanduvi, y Marcelo Giugale. 2011. Do our Children Have a Chance? A Human Opportunity Report for Latin America and the Caribbean. The World Bank.

Mora, Claudia. 2019. “Interseccionalidad y Fronteras Sociales: Género y Clase Social en el Mercado Laboral Chileno". Estudios Públicos (156): 47-67.

Múnera, Alfonso. 2005. Fronteras Imaginadas. Colombia: Editorial Planeta Colombiana.

Nuru-Jeter, Amani M., Elizabeth K. Michaels, Marilyn D. Thomas, Alexis N. Reeves, Roly J. Thorpe, y Thomas A. LaVeist. 2018. "Relative Roles of Race Versus Socioeconomic Position in Studies of Health Inequalities: A Matter of Interpretation." Annual Review of Public Health 39(1): 169-88.

Oaxaca, Ronald. 1973. "Male-Female Wage Differentials in Urban Labor Markets". International Economic Review 14(3): 693-709.

Otero-Bahamón, Silvia. 2016. When the State Minds the Gap: The Politics of Subnational Inequality in Latin America. PhD Thesis. Northwestern University.

Otero.Bahamón, Silvia. 2019. "Subnational Inequality in Latin America: Empirical and Theoretical Implications of Moving Beyond Interpersonal Inequality". Studies in Comparative International Development 54(2): 185-209.

Otero.Bahamón, Silvia. 2020. "Place-Sensitive Policies in the Provision of Subnational Public Goods in Colombia". Latin American Politics and Society 62(3): 94-122.

Programa de las Naciones Unidas para el Desarrollo PNUD. 2019. Informe Sobre Desarrollo Humano 2019. Nueva York: PNUD.

República de Chile. Instituto Nacional de Estadística INE. 2002. XVII Censo Nacional de Población y VI de Vivienda. Chile: INE.

República de Chile. Instituto Nacional de Estadística INE. 2017. Censo de Población y Vivienda 2017. Chile: INE.

República de Colombia. Departamento Administrativo Nacional de Estadística DANE. 2005. Censo General de Población 2005. Colombia: DANE

República de Colombia. Departamento Administrativo Nacional de Estadística DANE. 2018. Censo General de Población 2018. Colombia: DANE

República de Perú. Instituto Nacional de Estadística e Informática INEI. 2007. Censos Nacionales 2007: XI de Población y VI de Vivienda. Perú: INEI. 
República de Perú. Instituto Nacional de Estadística e Informática INEI. 2017. Censos Nacionales 2017: XII de Población y VII de Vivienda. Perú: INEI.

Rodó-de-Zárate, Maria, y Mireia Baylina. 2018. "Intersectionality in Feminist Geographies". Gender, Place \& Culture 25(4): 547-53.

Rogers, Melissa. 2021. "Federalism and the Welfare State in Latin America". Regional \& Federal Studies 31(1): 163-84.

Rosenthal, Lisa. 2016. "Incorporating Intersectionality Into Psychology: An Opportunity to Promote Social Justice and Equity." American Psychologist 71(6): 474-485.

Scott, Nick, y Janet Siltanen. 2012. "Gender and Intersectionality-A Quantitative Toolkit for Analyzing Complex Inequalities". Employment and Social Development Canada. Recu-

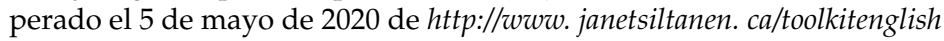

Sen, Bisakha. 2014. "Using the Oaxaca-Blinder Decomposition as an Empirical Tool to Analyze Racial Disparities in Obesity." Obesity 22 (7): 1750-55.

Shannon, Geordan D, Angelica Motta, Carlos F. Cáceres, Jolene Skordis-Worrall, Diana Bowie, y Audrey Prost. 2017. “ ¿Somos iguales? Using a Structural Violence Framework to Understand Gender and Health Inequities from an Intersectional Perspective in the Peruvian Amazon". Global Health Action 10(sup2): 43-45.

Singh, Prerna. 2016. How Solidarity Works for Welfare: Subnationalism and Social Development in India. New York: Cambridge University Press.

Spierings, Niels. 2016. "Multilevel Analysis as a Tool to Understand the Spatio-Temporality of Gender." Politics \& Gender 12(3) E10.

Solís, Patricio, Braulio Güémez Graniel, y Virginia Lorenzo Holm. 2019. Por Mi Raza Hablará La Desigualdad. Efectos de Las Características Étnico-Raciales En La Desigualdad de Oportunidades En México. México: Oxfam.

Thorp, Rosemary, y Maritza Paredes. 2010. Ethnicity and the Persistence of Inequality: The Case of Peru. Cham: Springer.

Vágvölgyi, Réka, Andra Coldea, Thomas Dresler, Josef Schrader, y Hans-Cristoph Nuerk. 2016. "A Review About Functional Illiteracy: Definition, Cognitive, Linguistic, and Numerical Aspects". Frontiers in Psychology 10.

Wade, Peter. 1997. Race and Ethnicity In Latin America. Pluto Press.

Recibido: 4 de agosto de 2020

Aceptado: 11 de marzo de 2021

Silvia Otero-Bahamón es profesora de la Facultad de Estudios Internacionales, Políticos y Urbanos de la Universidad del Rosario, en Bogotá, Colombia. Culminó su doctorado en Ciencia Política en Northwestern University en 2016, y una maestría en la misma institución (2013). Sus temas de investigación incluyen la política social, la economía política, la política comparada de América Latina, la formación del estado y los métodos cualitativos. En concreto, su agenda de investigación se centra en las dimensiones subnacionales de la desigualdad, sobre lo cual escribe actualmente un libro sobre la desigualdad social subnacional en América Latina y adelanta un proyecto de investigación sobre la reducción de la desigualdad de ingresos en cuatro ciudades colombianas. Ha publicado sus estudios en World Development, Latin American Politics and Society, Studies in Comparative International Development, Revista de Estudios Sociales, entre otras revistas. Es politóloga de la Universidad de los Andes (Bogotá, Colombia).

Correo electrónico: silvia.otero@urosario.edu.co 


\section{APÉNDICE}

En los métodos cuantitativos, el tratamiento más común del género, la etnia, y el lugar de residencia en los modelos de regresión lineal tradicional sigue siendo incluir estas categorías sociales como variables de control, en donde se "aisla" el efecto de cada una de estas categorías y se identifica qué tanto influencian la variable dependiente cuando se mantiene constante todo lo demás. Esto dista de ajustarse al enfoque interseccional porque cada categoría se concibe como "independiente, distinta, mutuamente excluyente", y el efecto de la variable de interés sobre el desenlace es el mismo entre distintas razas y géneros. El tratamiento de la variable de control en la regresión lineal tradicional responde a una lógica aditiva y no multiplicativa porque las desigualdades funcionan como capas superpuestas, en donde cada atributo o marcador de identidad añade una capa de ventaja y desventaja de la misma forma para todos los grupos (Scott y Siltanen 2012, Else-Quest y Hyde 2016).

En contraste, metodologías cuantitativas que toman en serio las premisas del enfoque interseccional parten de una noción multiplicativa de la desigualdad, en la que se consideran no sólo los efectos de cada uno de los atributos que componen la intersección sino que se identifica el "riesgo excesivo", o el riesgo que excede la suma del riesgo de los atributos individuales (Nuru-Jeter et al. 2018). Uno de los métodos preferidos para lograr esto es la inclusión de interacciones en los modelos de regresión. Esta metodología permite determinar cómo el impacto de una variable explicativa $\left(X_{1}\right)$ en una variable dependiente (Y) cambia como resultado de la variación en una tercera variable $\left(\mathrm{X}_{2}\right)$ (Scott y Siltanen 2012).

Si bien la introducción de interacciones en modelos de regresión es una de las apuestas cuantitativas preferidas, este método tiene varios problemas para materializar el enfoque interseccional. Primero, las interacciones entre dos categorías son fáciles de realizar e interpretar, pero la introducción de tres o más elementos en la interacción conlleva a modelos estadísticos más complejos y con menos poder estadístico. Con cada variable nueva incluida se disminuyen los tamaños de las muestras y la posibilidad de capturar significancia en la interacción, esto descontando la enorme dificultad de hacer una correcta interpretación de los resultados. Segundo, las intersecciones en modelos estadísticos no abandonan la noción aditiva de la desigualdad, y de hecho tienden a sobre-dimensionar los efectos aditivos y minimizar los efectos multiplicativos. Si el efecto de cada uno de las categorías sobre la variable dependiente es importante, el efecto de la intersección es necesariamente menor. Es decir que los efectos de la interacción dependen del tamaño de los efectos de cada variable por separado. De este modo, si el efecto de las categorías por separado es bajo, la intersección probablemente no será significativa (Bowleg 2012). 
